\title{
THE CALDERÓN PROBLEM WITH CORRUPTED DATA
}

\author{
PEDRO CARO AND ANDONI GARCIA
}

\begin{abstract}
We consider the inverse Calderón problem consisting of determining the conductivity inside a medium by electrical measurements on its surface. Ideally, these measurements determine the Dirichlet-to-Neumann map and, therefore, one usually assumes the data to be given by such map. This situation corresponds to having access to infinite-precision measurements, which is totally unrealistic. In this paper, we study the Calderón problem assuming the data to contain measurement errors and provide formulas to reconstruct the conductivity and its normal derivative on the surface. Additionally, we state the rate convergence of the method. Our approach is theoretical and has a stochastic flavour.
\end{abstract}

\section{INTRODUCTION}

In 1980, Calderón 11 proposed the following inverse boundary value problem: Let $D$ be a bounded domain in $\mathbb{R}^{d}(d \geq 2)$ with Lipschitz boundary $\partial D$, and let $\gamma$ be a real bounded measurable function in $D$ with a positive lower bound $\gamma_{0}$. Consider the linear map $\Lambda_{\gamma}: H^{1 / 2}(\partial D) \rightarrow H^{-1 / 2}(\partial D)$ defined-in a weak sense-by

$$
\Lambda_{\gamma} f=\left.\gamma \partial_{\nu} u\right|_{\partial D}
$$

where $\partial_{\nu}=\nu \cdot \nabla$, with $\nu$ denoting the outward unit normal vector to $\partial D$, and $u \in H^{1}(D)$ is the solution of the boundary value problem

$$
\left\{\begin{aligned}
\nabla \cdot(\gamma \nabla u) & =0 \text { in } D, \\
\left.u\right|_{\partial D} & =f .
\end{aligned}\right.
$$

In the literature, $\Lambda_{\gamma}$ is referred as the Dirichlet-to-Neumann map associated to $\gamma$ (DN map for short). The inverse problem is to decide whether $\gamma$ is uniquely determined by $\Lambda_{\gamma}$, and to calculate $\gamma$ in terms of $\Lambda_{\gamma}$ if $\gamma$ is indeed determined by $\Lambda_{\gamma}$.

This problem originates in electrical prospecting. If $D$ represents an inhomogeneous conductive medium with conductivity $\gamma$, the inverse Calderón problem is to determine the conductivity $\gamma$ in $D$ by means of steady state electrical measurements carried out on the surface of $D$. In this physical situation, $f$ represents the electric potential on the surface and $\Lambda_{\gamma} f$ represents the normal component of the outgoing electric current density on the surface. Ideally, $\Lambda_{\gamma}$ is determined through measurements effected on $\partial D$.

Implementing the theoretical results of the Calderón problem presents several non-trivial challenges. This is because theoretically one assumes to have access to infinite-precision measurements and to infinite many pieces of data, corresponding to knowing the whole graph of the DN map. Neither of these assumptions are justified in practice. On the one hand, only a finite number of measurements can be made to obtain our data. On the other hand, the data obtained will be corrupted by measurement errors and so they will not even lie on the graph of the DN map. The objective of this paper will be to address the question of data corruption in the Calderón problem. For this purpose, we assume data to be given

Date: January 9, 2017. 
by points on the graph of the DN map plus an error modelled by random white noise. In mathematical terms, we consider a complete probability space $(\Omega, \mathcal{H}, \mathbb{P})$, and a countable family $\left\{X_{\alpha}: \alpha \in \mathbb{N}^{2}\right\}$ of independent complex Gaussian random variables $X_{\alpha}: \omega \in \Omega \mapsto X_{\alpha}(\omega) \in \mathbb{C}$ such that

$$
\mathbb{E} X_{\alpha}=0, \quad \mathbb{E}\left(X_{\alpha} \overline{X_{\alpha}}\right)=1, \quad \mathbb{E}\left(X_{\alpha} X_{\alpha}\right)=0 \quad \forall \alpha \in \mathbb{N}^{2} .
$$

We adopt the standard notation for the expectation of a random variable $X$ :

$$
\mathbb{E} X=\int_{\Omega} X d \mathbb{P}
$$

Then, we propose to define the noisy data for the Calderón problem as the bilinear form

$$
\mathcal{N}_{\gamma}(f, g)=\int_{\partial D} \Lambda_{\gamma} f g+\sum_{\alpha \in \mathbb{N}^{2}}\left(f \mid e_{\alpha_{1}}\right)\left(g \mid e_{\alpha_{2}}\right) X_{\alpha} \quad \forall f, g \in H^{1 / 2}(\partial D)
$$

where $\alpha=\left(\alpha_{1}, \alpha_{2}\right),\left\{e_{n}: n \in \mathbb{N}\right\}$ is an orthonormal basis of $L^{2}(\partial D)$ and $(\phi \mid \psi)=$ $\int_{\partial D} \phi \bar{\psi}$. 1 We will see, in the corollary 2.4 below, that $\mathcal{N}_{\gamma}(f, g) \in L^{2}(\Omega, \mathcal{H}, \mathbb{P})$, and consequently $\left|\mathcal{N}_{\gamma}(f, g)\right|<\infty$ almost surely. Note that

$$
\mathbb{E} \mathcal{N}_{\gamma}(f, g)=\int_{\partial D} \Lambda_{\gamma} f g
$$

which corresponds to saying that, with access to many independent outcomes $\left\{\mathcal{N}_{\gamma}(f, g)\left(\omega_{n}\right): n \in \mathbb{N}\right\}$, we can filter out the noise by averaging

$$
\frac{1}{N} \sum_{n=0}^{N-1} \mathcal{N}_{\gamma}(f, g)\left(\omega_{n}\right) \underset{N \rightarrow \infty}{\longrightarrow} \int_{\partial D} \Lambda_{\gamma} f g .
$$

In practice, repetitions of the same measurement do not oscillate enough to be filtered out by averaging. Therefore, our objective should avoid averaging different realizations and show that a single realization of $\mathcal{N}_{\gamma}(f, g)$ is enough to reconstruct $\gamma$.

Problem. Assuming $\gamma$ and $\partial D$ to be as smooth as needed, show that $\gamma$ can be calculated from $\mathcal{N}_{\gamma}$ almost surely.

The question of how to model the noise in inverse problems is of capital importance, since infinite-precision measurements are totally unrealistic. There seem to be two different approaches: one based on deterministic regularization techniques, assuming the noise to be deterministic and small [31, 32; and another based on a statistical point of view [28, 16], which does not need to assume smallness of the noise. See also the works [20, 21]. Knudsen, Lassas, Mueller and Siltanen [22] used regularization techniques to study the Calderón problem in dimension $d=2$ with noisy data. In order to carry out their deterministic analysis, they assumed the noise level to be small. Our approach has a stochastic flavour with no restriction on the size of the noise. In the context of the Calderón problem, this seems to be a new approach. In this paper we show that $\left.\gamma\right|_{\partial D}$ and $\left.\partial_{\nu} \gamma\right|_{\partial D}$ can be reconstructed from a single realization of $\mathcal{N}_{\gamma}$.

Theorem 1. Let $D$ be a bounded domain of $\mathbb{R}^{d}(d \geq 2)$ with Lipschitz boundary $\partial D$. Consider $\gamma$ a Lipschitz continuous conductivity in $\bar{D}$. Then, for almost every $P \in \partial D$, there exists an explicit sequence $\left\{f_{N}: N \in \mathbb{N} \backslash\{0\}\right\}$ in $H^{1 / 2}(\partial D)$ such that

almost surely.

$$
\lim _{N \rightarrow \infty} \mathcal{N}_{\gamma}\left(f_{N}, \overline{f_{N}}\right)=\gamma(P)
$$

\footnotetext{
1 The first integral on the definition of $\mathcal{N}_{\gamma}$ is an abuse of notation, in fact meaning the duality pairing between $H^{1 / 2}(\partial D)$ and $H^{-1 / 2}(\partial D)$.
} 
Our theorem only establishes a reconstruction procedure for almost every $P$ in $\partial D$. However, in the proposition 2.7 we describe the set of boundary points for which the reconstruction algorithm works. It is worth to point out that this description only requires local smoothness of $\partial D$. In fact, if the domain was $C^{1}$ the theorem would hold for every point $P \in \partial D$.

The theorem 1 extends a result with ideal data due to Brown [6] for the particular case that $\gamma$ is Lipschitz - Brown's theorem holds for very low regular conductivities. We believe that our theorem also holds at the same level of regularity with no extra effort.

The rate convergence of the limit in the theorem 1 is described in the next theorem.

Theorem 2. Let $D$ be a bounded domain of $\mathbb{R}^{d}(d \geq 2)$ with a $C^{1, \theta}$ boundary $\partial D$ for $0<\theta<1$. Consider $\gamma$ as in the theorem 1 . Then, for every $P \in \partial D$, there exist an explicit sequence $\left\{f_{N}: N \in \mathbb{N} \backslash\{0\}\right\}$ in $H^{1 / 2}(\partial D)$ and a constant $C>0$ (depending on $d, \partial D$, a lower bound on $\gamma_{0}$ and an upper bound for $\|\gamma\|_{C^{0,1}(\bar{D})}$ ) such that, for every $\epsilon>0$, we have

$$
\mathbb{P}\left\{\left|\mathcal{N}_{\gamma}\left(f_{N}, \overline{f_{N}}\right)-\gamma(P)\right| \leq C N^{-\theta /(1+\theta)}\right\} \geq 1-\epsilon \quad \forall N \geq c \epsilon^{-\frac{1+\theta}{1-\theta}} .
$$

Here $c$ only depends on $\partial D$ and $\theta$.

In this theorem, the regularity of $\gamma$ could have been lowered to $C^{0, \theta}$ with no extra effort and no loss on the rate of convergence. However, in order to get a rate of convergence of the type stated in our theorem, the method requires Hölder continuity for the conductivity and the first derivatives of the functions describing locally the boundary of $D$. We believe that this a priori regularity is also required when having ideal data. However, the stability of the problem for ideal data is Lipschitz under the assumptions of Brown's theorem [17]. This seems to tell that even if a reconstruction method provides Lipschitz stability for the problem, the rate of convergence of the same method could be worse or require extra assumptions.

In the next theorem we provide a formula to reconstruct the normal derivative of the conductivity at the boundary, once we know the conductivity at the boundary. For this, we will use a reference medium with an homogeneous conductivity identically one. Its corresponding DN map will be denoted by $\Lambda$.

Theorem 3. Let $D$ be a bounded domain of $\mathbb{R}^{d}(d \geq 2)$ with $C^{1,1}$ boundary $\partial D$ and assume $\gamma \in C^{1,1}(\bar{D})$. Then, for every $P \in \partial D$, there exists an explicit family $\left\{f_{t}: t \geq 1\right\}$ in $H^{1 / 2}(\partial D)$ such that

$$
\lim _{N \rightarrow \infty} \frac{1}{T_{N}} \int_{T_{N}}^{2 T_{N}}\left[\mathcal{N}_{\gamma}\left(f_{t^{2}}, \overline{f_{t^{2}}} / \gamma\right)-\int_{\partial D} \Lambda f_{t^{2}} \overline{f_{t^{2}}}\right] d t=\frac{\partial_{\nu_{P}} \gamma(P)+i \tau_{P} \cdot \nabla \gamma(P)}{\gamma(P)}
$$

almost surely where $N \in \mathbb{N} \backslash\{0\}$ and $T_{N}=N^{3+3 \theta / 2}$ with $\theta \in(0,1)$. Here $\nu_{P}$ is the outward unit normal vector to $\partial D$ at $P$ and $\tau_{P}$ denotes any unitary tangential vector at $P$.

Brown and Salo [8] proved a similar result to ours for the steady state heat equation with convection assuming access to infinite-precision data. Their result could be rewritten, in the case of ideal data, for the conductivity equation assuming $\gamma$ and $\partial D$ to be $C^{1}$. Our theorem 3 extends this for the particular case where $\gamma$ and $\partial D$ are $C^{1,1}$. In this case, our method fails for less regular assumptions - see the lemma 3.5 below. In the appendix of [17], Brown in collaboration with García and Zhang proved that the normal derivative of the conductivity on the boundary can be recovered from ideal data assuming the boundary to be Lipschitz. This approach does not seem to be so convenient for our case since the formula is non-linear with 
respect to the data (see the theorem 7 in [17]) and this may cause difficulties when filtering out the noise.

Our last theorem describes the rate of convergence of the limit in the previous theorem.

Theorem 4. Let $D$ be a bounded domain of $\mathbb{R}^{d}(d \geq 2)$ with $C^{1,1}$ boundary $\partial D$ and assume $\gamma \in C^{1,1}(\bar{D})$. Consider $P \in \partial D$ and $\left\{f_{t}: t \geq 1\right\}$ the family of the theorem 3. For every $N \in \mathbb{N} \backslash\{0\}$, set

$$
Y_{N}=\frac{1}{T_{N}} \int_{T_{N}}^{2 T_{N}}\left[\mathcal{N}_{\gamma}\left(f_{t^{2}}, \overline{f_{t^{2}}} / \gamma\right)-\int_{\partial D} \Lambda f_{t^{2}} \overline{f_{t^{2}}}\right] d t
$$

with $T_{N}$ as in the theorem 3. Then, there exists a constant $C>0$ (depending on $d$, $\partial D$, a lower bound on $\gamma_{0}$ and an upper bound for $\left.\|\gamma\|_{C^{1,1}(\bar{D})}\right)$ such that, for every $\epsilon>0$, we have

$$
\mathbb{P}\left\{\left|Y_{N}-\frac{\partial_{\nu_{P}} \gamma(P)+i \tau_{P} \cdot \nabla \gamma(P)}{\gamma(P)}\right| \leq C N^{-\theta}\right\} \geq 1-\epsilon \quad \forall N>c \epsilon^{-\frac{1}{1-\theta}} .
$$

Here $c$ depends on $\theta, d, \partial D$, a lower bound for $\gamma_{0}$ and an upper bound for $\|\gamma\|_{C^{1,1}(\bar{D})}$.

The noise have been assumed to be Gaussian, however, in this paper this is not required. The reason for us to define the noise as Gaussian is because we believe that this will be convenient for the reconstruction of $\gamma$ in the interior of $D$.

To prove these theorems, we use the family of solutions constructed by Brown and Salo in the papers [6, 8]. Our main contribution consists of noting that these solutions are robust enough to get rid of the error measurement by making an appropriate averaging on the parameter of the family. For the theorems 1 and 2 this is not even required because $\left\|f_{N}\right\|_{L^{2}(\partial D)}=\mathcal{O}\left(N^{-1 / 2}\right)$. However, for the theorems 3 and 4 we only have that $\left\|f_{N}\right\|_{L^{2}(\partial D)}=\mathcal{O}(1)$, which makes necessary the average in $\sqrt{N}$. This could be thought as an ergodic property of the traces of this family when applied to the noisy data. In other words, the noisy data generated by this family of solutions is statistically stable. We hope this paper could inspire a different way of dealing with noise in the numerical reconstruction of the conductivity.

The classical references for the Calderón problem with full ideal data and isotropic conductivities are [23, 24] due to Kohn and Vogelius, global uniqueness for $d \geq 3$ 29] by Silvester and Uhlmann, the work of stability [1] due to Alessandrini, reconstruction by Nachman [25] and uniqueness in $d=2$ [26] due to Nachman. See also [30, 2]. More recent references dealing with questions of regularity in dimension $d=2$ are [10, 3] for uniqueness and [4, 5, 14, for stability. For the uniqueness in dimension $d \geq 3$ [7, 9, 27, 19, 18, 13, the stability [12] and the reconstruction [17].

Regarding stochastic approaches, Dunlop and Stuart have recently given a rigorous Bayesian formulation of the electrical impedance tomography problem [15].

Our paper contains other two sections. In the first one, we prove the theorems 1 and 2. The second one is devoted to the theorems 3 and 4

\section{ReCOVERING THE CONDUCTIVITY AT THE BOUNDARY}

In this section we prove the theorems 1 and 2 . Here we assume $\gamma \in C^{0,1}(\bar{D})$ with $\gamma(x) \geq \gamma_{0}>0$ for all $x \in \bar{D}$ and the boundary of $D$ to be represented locally by the graphs of some Lipschitz functions. Thus, for each $P \in \partial D$, there is a coordinate system $\left(y^{\prime}, y_{d}\right) \in \mathbb{R}^{d-1} \times \mathbb{R}$, a constant $\rho>0$ and a Lipschitz function $\phi: \mathbb{R}^{d-1} \rightarrow \mathbb{R}$ so that

$$
B(P, \rho) \cap D=B(P, \rho) \cap\left\{y \in \mathbb{R}^{d}: y_{d}>\phi\left(y^{\prime}\right)\right\}
$$


and

$$
B(P, \rho) \cap \partial D=B(P, \rho) \cap\left\{y \in \mathbb{R}^{d}: y_{d}=\phi\left(y^{\prime}\right)\right\} .
$$

Let $\left(p^{\prime}, \phi\left(p^{\prime}\right)\right)$ denote the coordinates of $P$ in the corresponding system and $F$ the map $F(x)=\left(x^{\prime}+p^{\prime}, x_{d}+\phi\left(x^{\prime}+p^{\prime}\right)\right)$. Let $\tilde{D}$ denote the pre-image of $D$ under $F$, that is $\tilde{D}=F^{-1}(D)$.

Before going further, we observe that if $u$ solves the problem (1), then $F^{*} u(x)=$ $u(F(x))$ solves the equation $\nabla \cdot\left(A_{\gamma}(x) \nabla F^{*} u\right)=0$ in $\tilde{D}$, where

$$
A_{\gamma}(x)=\gamma(F(x)) \nabla F^{-1}(F(x)) \nabla F^{-1}(F(x))^{t},
$$

where $\nabla F^{-1}(y)^{t}$ is the transpose of

$$
\nabla F^{-1}(y)=\left[\begin{array}{cc}
I_{d-1} & 0 \\
-\nabla \phi\left(y^{\prime}\right) & 1
\end{array}\right]
$$

with $I_{d-1}$ the identity in $d-1$. Furthermore, since the Jacobian $|\operatorname{det} \nabla F(x)|=1$, we have that

$$
\begin{aligned}
\int_{\partial D} \Lambda_{\gamma} f \bar{f} & =\int_{D} \gamma(y)|\nabla u(y)|^{2} d y \\
& =\int_{\tilde{D}} \gamma(F(x)) \nabla u(F(x)) \cdot \nabla \bar{u}(F(x))|\operatorname{det} \nabla F(x)| d x \\
& =\int_{\tilde{D}} \nabla F^{*} u(x) \cdot\left(A_{\gamma}(x) \nabla F^{*} \bar{u}(x)\right) d x .
\end{aligned}
$$

We let $\eta: \mathbb{R} \rightarrow[0,1]$ to be a smooth function which satisfies $\eta(t)=1,|t| \leq 1 / 2$ and $\eta(t)=0,|t| \geq 1$. We choose $\xi \in \mathbb{R}^{d} \backslash\{0\}$ a constant vector for which $\xi \cdot A_{\gamma}(0) \xi=$ $e_{d} \cdot A_{\gamma}(0) e_{d}$ and $\xi \cdot A_{\gamma}(0) e_{d}=0$. Note that this choice makes $\xi=\left(\xi^{\prime}, \xi_{d}\right)$ satisfy $\left|\xi^{\prime}\right| \neq 0$. For $N \geq M \geq 1$, we set

$$
a_{M, N}(x)=\eta\left(M\left|x^{\prime}\right|\right) \eta\left(M x_{d}\right) e^{N\left(i \xi-e_{d}\right) \cdot x}=\chi(M x) e^{N\left(i \xi-e_{d}\right) \cdot x},
$$

where the function $\chi(x)=\eta\left(\left|x^{\prime}\right|\right) \eta\left(x_{d}\right)$ has been introduced to simplify notation. The lemmas 1 and 2 in [6] can be written in our particular case as follows:

Lemma 2.1 (Brown [6]). If $\gamma \in C^{0,1}(\bar{D}), A_{\gamma}$ is defined as in (4) and $\nabla \phi\left(p^{\prime}\right)$ exists. Then we have

$$
\begin{aligned}
\int_{\tilde{D}} \nabla a_{M, N}(x) \cdot & \left(A_{\gamma}(x) \nabla \overline{a_{M, N}}(x)\right) d x=N M^{1-d} \gamma(P)\left(1+\left|\nabla \phi\left(p^{\prime}\right)\right|^{2}\right) \int_{\mathbb{R}^{d-1}} \eta\left(\left|x^{\prime}\right|\right)^{2} d x^{\prime} \\
& +\mathcal{O}\left(M^{2-d}+N M^{-d}+N \int_{\left|x^{\prime}\right| \leq M^{-1}}\left|\nabla \phi\left(x^{\prime}+p^{\prime}\right)-\nabla \phi\left(p^{\prime}\right)\right| d x^{\prime}\right) .
\end{aligned}
$$

The constant implicit in $\mathcal{O}$ depends on $d$ and on upper bounds for $\|\gamma\|_{C^{0,1}(\bar{D})}$ and $\|\phi\|_{C^{0,1}\left(\mathbb{R}^{d-1}\right)}$.

Lemma 2.2 (Brown [6]). Consider $a_{M, N}$ as in (6) and let $w_{M, N}$ solve the boundary value problem

$$
\left\{\begin{aligned}
\nabla \cdot\left(A_{\gamma} \nabla w_{M, N}\right) & =-\nabla \cdot\left(A_{\gamma} \nabla a_{M, N}\right) \text { in } \tilde{D}, \\
\left.w_{M, N}\right|_{\partial \tilde{D}} & =0 .
\end{aligned}\right.
$$

If $\nabla \phi\left(p^{\prime}\right)$ exists, then

$\left\|\nabla w_{M, N}\right\|_{L^{2}(\tilde{D})} \lesssim N^{-\frac{1}{2}} M^{\frac{3-d}{2}}+M^{\frac{1-d}{2}}+N^{\frac{1}{2}}\left(\int_{\left|x^{\prime}\right| \leq M^{-1}}\left|\nabla \phi\left(x^{\prime}+p^{\prime}\right)-\nabla \phi\left(p^{\prime}\right)\right|^{2} d x^{\prime}\right)^{\frac{1}{2}}$

where the implicit constant depends on $d$, a lower bound on $\gamma_{0}$ and on upper bounds for $\|\gamma\|_{C^{0,1}(\bar{D})}$ and $\|\phi\|_{C^{0,1}\left(\mathbb{R}^{d-1}\right)}$. 
Following [6], we choose $M=N^{1 / 2}$ and consider the function $u_{N}$ defined by

$$
F^{*} u_{N}=N^{-\frac{1}{2}} M^{\frac{d-1}{2}} C_{P}\left(a_{M, N}+w_{M, N}\right)
$$

with $C_{P}=\left(\left(1+\left|\nabla \phi\left(p^{\prime}\right)\right|^{2}\right) \int_{\mathbb{R}^{d-1}} \eta\left(\left|x^{\prime}\right|\right)^{2} d x^{\prime}\right)^{-1 / 2}$ and $f_{N}=\left.u_{N}\right|_{\partial D}$. Note that after (3), (5) and the lemmas 2.1 and 2.2 we have

$$
\begin{aligned}
\mathcal{N}_{\gamma}\left(f_{N}, \overline{f_{N}}\right) & =\int_{\tilde{D}} \nabla F^{*} u_{N}(x) \cdot\left(A_{\gamma}(x) \nabla F^{*} \overline{u_{N}}(x)\right) d x+\sum_{\alpha \in \mathbb{N}^{2}}\left(f_{N} \mid e_{\alpha_{1}}\right)\left(\overline{f_{N}} \mid e_{\alpha_{2}}\right) X_{\alpha} \\
& =\gamma(P)+\sum_{\alpha \in \mathbb{N}^{2}}\left(f_{N} \mid e_{\alpha_{1}}\right)\left(\overline{f_{N}} \mid e_{\alpha_{2}}\right) X_{\alpha}+\mathcal{O}\left(N^{-\frac{1}{2}}+h(M)\right),
\end{aligned}
$$

where

$$
h(M)=\left(M^{d-1} \int_{\left|x^{\prime}\right| \leq M^{-1}}\left|\nabla \phi\left(x^{\prime}+p^{\prime}\right)-\nabla \phi\left(p^{\prime}\right)\right|^{2} d x^{\prime}\right)^{\frac{1}{2}} .
$$

In the following lines, we will show that $\mathcal{N}_{\gamma}\left(f_{N}, \overline{f_{N}}\right)$ tends to $\gamma(P)$ as $N$ goes to infinity. The last term will vanish, in the limit, under appropriate assumptions on $\partial D$. To show that the second vanishes almost surely in the limit, we will use a very simple idea of Lebesgue spaces - see the lemma 2.5 below. Before this let us make some comments about $\mathcal{N}_{\gamma}$.

Lemma 2.3. There exists a complete probability space $(\Omega, \mathcal{H}, \mathbb{P})$, and a countable family $\left\{X_{\alpha}: \alpha \in \mathbb{N}^{2}\right\}$ of independent complex random variables satisfying (2). Moreover, for every $f, g \in L^{2}(\partial D)$ we have that

$$
\mathbb{E}\left|\sum_{\alpha \in \mathbb{N}^{2}}\left(f \mid e_{\alpha_{1}}\right)\left(g \mid e_{\alpha_{2}}\right) X_{\alpha}\right|^{2}=\|f\|_{L^{2}(\partial D)}^{2}\|g\|_{L^{2}(\partial D)}^{2} .
$$

Proof. The existence part is a consequence of for example Ionescu-Tulcea's theorem. The second part is a simple consequence of the independence of $\left\{X_{\alpha}: \alpha \in \mathbb{N}^{2}\right\}$ and the facts $\mathbb{E}\left(X_{\alpha} \overline{X_{\alpha}}\right)=1$ for all $\alpha \in \mathbb{N}^{2}$ and that $\left\{e_{n}: n \in \mathbb{N}\right\}$ is an orthonormal basis of $L^{2}(\partial D)$.

Corollary 2.4. The corrupted data

$$
\mathcal{N}_{\gamma}: H^{1 / 2}(\partial D) \times H^{1 / 2}(\partial D) \rightarrow L^{2}(\Omega, \mathcal{H}, P)
$$

is bounded in the sense that, there exists a constant $C>0$ depending on $d$ and $\partial D$ such that

$$
\mathbb{E}\left|\mathcal{N}_{\gamma}(f, g)\right|^{2} \leq C\left(1+\|\gamma\|_{L^{\infty}(D)}^{2}\right)\|f\|_{H^{1 / 2}(\partial D)}^{2}\|g\|_{H^{1 / 2}(\partial D)}^{2}
$$

for all $f, g \in H^{1 / 2}(\partial D)$. Consequently, $\left|\mathcal{N}_{\gamma}(f, g)\right|<\infty$ almost surely.

As a consequence of the lemma 2.3

$$
\mathbb{E}\left|\sum_{\alpha \in \mathbb{N}^{2}}\left(f_{N} \mid e_{\alpha_{1}}\right)\left(\overline{f_{N}} \mid e_{\alpha_{2}}\right) X_{\alpha}\right|^{2}=\left\|f_{N}\right\|_{L^{2}(\partial D)}^{4} .
$$

On the other hand, a simple computation shows that

$$
\left\|f_{N}\right\|_{L^{2}(\partial D)}^{2} \leq C_{\partial D} N^{-1}
$$

where the constant $C_{\partial D}>0$ only depends on an upper bound for $\|\phi\|_{C^{0,1}\left(\mathbb{R}^{d-1}\right)}$.

The rest of argument relies on the following lemma.

Lemma 2.5. Let $(X, \Sigma, \mu)$ be a measure space and $\left\{f_{n}: n \in \mathbb{N}\right\}$ be a sequence in $L^{p}(X, \Sigma, \mu)$ and $f \in L^{p}(X, \Sigma, \mu)$ with $1 \leq p<\infty$ such that

$$
f_{n} \rightarrow f \quad \text { in } L^{p}(X, \Sigma, \mu),
$$


as $n \rightarrow \infty$. Assume that there exists a sequence $\left\{\lambda_{n}: n \in \mathbb{N}\right\}$ of positive real numbers such that $\lambda_{n} \rightarrow 0$ as $n \rightarrow \infty$ and

$$
\sum_{n \in \mathbb{N}} \frac{1}{\lambda_{n}^{p}} \int_{X}\left|f_{n}-f\right|^{p} d \mu<\infty
$$

Then,

$$
f_{n}(x) \rightarrow f(x)
$$

as $n \rightarrow \infty$ for almost every $x \in X$.

Assume furthermore that $\mu(X)<\infty$. Then, for every $\epsilon>0$, there exists a $n_{0} \in \mathbb{N}$ such that

$$
\mu\left(\left\{x \in X:\left|f_{n}(x)-f(x)\right| \leq \lambda_{n}\right\}\right) \geq \mu(X)-\epsilon,
$$

for $n \geq n_{0}$.

Proof. The first part of this lemma follows from the fact that

$$
\mu\left(\bigcap_{n=1}^{\infty} \bigcup_{k=n}^{\infty} E_{k}\right)=0
$$

for $E_{n}=\left\{x \in X:\left|f_{n}(x)-f(x)\right|>\lambda_{n}\right\}$. Indeed, if $x \notin \cap_{n=1}^{\infty} \cup_{k=n}^{\infty} E_{k}$ there exists a $n_{x} \in \mathbb{N}$ such that $x \notin \cup_{k=n}^{\infty} E_{k}$ for all $n \geq n_{x}$, which means that

$$
\left|f_{n}(x)-f(x)\right|<\lambda_{n}, \quad \forall n \geq n_{x} .
$$

The identity $(9)$ holds as a consequence of the following inequalities

$$
\mu\left(\bigcap_{n=1}^{\infty} \bigcup_{k=n}^{\infty} E_{k}\right) \leq \sum_{k=n}^{\infty} \mu\left(E_{k}\right) \leq \sum_{k=n}^{\infty} \frac{1}{\lambda_{k}^{p}} \int_{X}\left|f_{k}-f\right|^{p} d \mu .
$$

To prove the second part, let $n_{0}$ be such that

$$
\sum_{n=n_{0}}^{\infty} \frac{1}{\lambda_{n}^{p}} \int_{X}\left|f_{n}-f\right|^{p} d \mu \leq \epsilon
$$

Then,

$$
\mu\left(X \backslash \bigcup_{n=n_{0}}^{\infty} E_{n}\right) \geq \mu(X)-\sum_{n=n_{0}}^{\infty} \mu\left(E_{n}\right) \geq \mu(X)-\epsilon
$$

where $x \in X \backslash \cup_{n=n_{0}}^{\infty} E_{n}$ if and only if $\left|f_{n}(x)-f(x)\right| \leq \lambda_{n}$ for all $n \geq n_{0}$.

Remark 2.6. Note that, given $\epsilon>0$, the $n_{0}$ stated in the lemma 2.5 only has to satisfy 10 .

Applying the first part of this lemma to $L^{2}(\Omega, \mathcal{H}, \mathbb{P})$, the sequence

$$
\sum_{\alpha \in \mathbb{N}^{2}}\left(f_{N} \mid e_{\alpha_{1}}\right)\left(\overline{f_{N}} \mid e_{\alpha_{2}}\right) X_{\alpha}
$$

and $\lambda_{N}=N^{-\theta}$ with $\theta \in(0,1 / 2)$, we have the following proposition:

Proposition 2.7. Let $P \in \partial D$ such that the corresponding boundary function $\phi$ satisfies

$$
\lim _{r \rightarrow 0} \frac{1}{r^{d-1}} \int_{\left|x^{\prime}\right| \leq r}\left|\nabla \phi\left(x^{\prime}+p^{\prime}\right)-\nabla \phi\left(p^{\prime}\right)\right|^{2} d x^{\prime}=0 .
$$

Then, if $N \in \mathbb{N} \backslash\{0\}$ we have that

$$
\lim _{N \rightarrow \infty} \mathcal{N}_{\gamma}\left(f_{N}, \overline{f_{N}}\right)=\gamma(P)
$$

almost surely. 
It is well known that, for almost every $P \in \partial D$, its corresponding boundary functions $\phi$ satisfies (11). Therefore, the theorem 1 holds.

The theorem 2 will be a consequence of the following proposition.

Proposition 2.8. Let $P \in \partial D$ such that the corresponding boundary function $\phi$ satisfies

$$
\left|\nabla \phi\left(x^{\prime}+p^{\prime}\right)-\nabla \phi\left(p^{\prime}\right)\right| \leq L\left|x^{\prime}\right|^{\theta}
$$

with $L>0$ and $0<\theta<1$. Consider $u_{N}$ as in (7) with $M=N^{1 /(1+\theta)}$ and $N \in \mathbb{N} \backslash\{0\}$. Then, there exists a constant $C>0$ (depending on $d$, a lower bound on $\gamma_{0}$ and on upper bounds for $\|\gamma\|_{C^{0,1}(\bar{D})},\|\phi\|_{C^{0,1}\left(\mathbb{R}^{d-1}\right)}$ and $\left.L\right)$ such that, for every $\epsilon>0$, we have

$$
\mathbb{P}\left\{\left|\mathcal{N}_{\gamma}\left(f_{N}, \overline{f_{N}}\right)-\gamma(P)\right| \leq C N^{-\theta /(1+\theta)}\right\} \geq 1-\epsilon \quad \forall N \geq c \epsilon^{-\frac{1+\theta}{1-\theta}} .
$$

Here $c$ only depends on $C_{\partial D}$ and $\theta$.

Proof. Using (3), (5), the lemmas 2.1 and 2.2 and 12 we can see that

$$
\begin{aligned}
\mathcal{N}_{\gamma}\left(f_{N}, \overline{f_{N}}\right) & =\int_{\tilde{D}} \nabla F^{*} u_{N}(x) \cdot\left(A_{\gamma}(x) \nabla F^{*} \overline{u_{N}}(x)\right) d x+\sum_{\alpha \in \mathbb{N}^{2}}\left(f_{N} \mid e_{\alpha_{1}}\right)\left(\overline{f_{N}} \mid e_{\alpha_{2}}\right) X_{\alpha} \\
& =\gamma(P)+\sum_{\alpha \in \mathbb{N}^{2}}\left(f_{N} \mid e_{\alpha_{1}}\right)\left(\overline{f_{N}} \mid e_{\alpha_{2}}\right) X_{\alpha}+\mathcal{O}\left(N^{-\frac{1}{2}}+M^{-\theta}\right) .
\end{aligned}
$$

Applying the second part of the lemma 2.5 for $L^{2}(\Omega, \mathcal{H}, \mathbb{P}), \lambda_{N}=N^{-\theta /(1+\theta)}$ and the sequence $\left\{\sum\left(f_{N} \mid e_{\alpha_{1}}\right)\left(\overline{f_{N}} \mid e_{\alpha_{2}}\right) X_{\alpha}: N \in \mathbb{N} \backslash\{0\}\right\}$, and using

$$
\mathbb{E}\left|\sum_{\alpha \in \mathbb{N}^{2}}\left(f_{N} \mid e_{\alpha_{1}}\right)\left(\overline{f_{N}} \mid e_{\alpha_{2}}\right) X_{\alpha}\right|^{2} \leq C_{\partial D^{2}}^{2} N^{-2}
$$

with $C_{\partial D}$ as in 8 , we know that:

$$
\mathbb{P}\left\{\left|\sum\left(f_{N} \mid e_{\alpha_{1}}\right)\left(\overline{f_{N}} \mid e_{\alpha_{2}}\right) X_{\alpha}\right| \leq N^{-\theta /(1+\theta)}\right\} \geq 1-\epsilon
$$

for every $N \geq N_{0}$. According to the remark 2.6 it is enough to choose $N_{0}>1$ satisfying

$$
\sum_{N=N_{0}}^{\infty} \frac{C_{\partial D}^{2}}{N^{\frac{2}{1+\theta}}} \leq \epsilon
$$

which holds whenever

$$
\frac{C_{\partial D}^{2}}{\epsilon} \frac{1+\theta}{1-\theta}<\left(N_{0}-1\right)^{\frac{1-\theta}{1+\theta}} .
$$

From the identity at the beginning of this proof and the choice $M=N^{1 /(1+\theta)}$, we see that there exists a constant $C>0$ such that

$\left\{\left|\sum\left(f_{N} \mid e_{\alpha_{1}}\right)\left(\overline{f_{N}} \mid e_{\alpha_{2}}\right) X_{\alpha}\right| \leq N^{-\theta /(1+\theta)}\right\} \subset\left\{\left|\mathcal{N}_{\gamma}\left(f_{N}, \overline{f_{N}}\right)-\gamma(P)\right| \leq C N^{-\theta /(1+\theta)}\right\}$

which is enough to conclude the proof.

If $D$ has a $C^{1, \theta}$ boundary, then every point $P \in \partial D$ satisfies $\sqrt{12}$, and consequently the theorem 2 holds. 


\section{The NORMAL DERIVATIVE OF THE CONDUCTIVITY AT THE BOUNDARY}

Here we prove the theorems 3 and 4 . We start by considering an integral identity that brings up the gradient of the conductivity.

Lemma 3.1. Let $u \in H^{1}(D)$ be the unique solution of the boundary value problem (1) and $v \in H^{1}(D)$ the harmonic extension in $D$ of $g \in H^{1 / 2}(\partial D)$. Then,

$$
\int_{\partial D}\left(\frac{1}{\gamma} \Lambda_{\gamma}-\Lambda\right) f g=-\int_{D} \frac{\nabla \gamma}{\gamma} \cdot \nabla u v
$$

where $\Lambda$ is the $D N$ map associated to the conductivity identically one.

Proof. By the definition of the DN map,

$$
\int_{\partial D} \frac{1}{\gamma} \Lambda_{\gamma} f g=\int_{D} \gamma \nabla u \cdot \nabla\left(\frac{v}{\gamma}\right)
$$

since $v / \gamma \in H^{1}(D)$ and $\left.v\right|_{\partial D}=g$. Furthermore, we have

$$
\int_{D} \gamma \nabla u \cdot \nabla\left(\frac{v}{\gamma}\right)=\int_{D} \nabla u \cdot \nabla v-\int_{D} \frac{\nabla \gamma}{\gamma} \cdot \nabla u v
$$

by the Leibniz rule. Consider $w \in H^{1}(D)$ the harmonic extension in $D$ of $f$. Adding and subtracting $\nabla w$ as appropriate we see that

$$
\int_{D} \nabla u \cdot \nabla v=\int_{D} \nabla(u-w) \cdot \nabla v+\int_{\partial D} \Lambda f g
$$

by the definition of $\Lambda$. Since $v$ is harmonic in $D$, the first term in the right hand side of the previous identity vanishes. Eventually, the integral identity we want to prove follows from the former considerations.

With this identity at hand, we just plug in $a_{M, N}$ as in $(6)$ in order to obtain an asymptotic equality similar to the one in the lemma 2.1 .

Lemma 3.2. Assume $\gamma \in C^{1,1}(\bar{D})$ and $\phi \in C^{1,1}\left(\mathbb{R}^{d-1}\right)$. Then we have

$$
\begin{aligned}
\int_{\tilde{D}} & \frac{\nabla \gamma(F(x))}{\gamma(F(x))} \cdot\left(\nabla F^{-1}(F(x))^{t} \nabla a_{M, N}(x)\right) \overline{a_{M, N}(x)} d x \\
= & M^{1-d} \frac{1}{2} \frac{\nabla \gamma(P)}{\gamma(P)} \cdot\left(\nabla F^{-1}(P)^{t}\left(i \xi-e_{d}\right)\right) \int_{\mathbb{R}^{d-1}} \eta\left(\left|x^{\prime}\right|\right)^{2} d x^{\prime} \\
& \quad+\mathcal{O}\left(M^{2-d} N^{-1}+M^{-d}\right) .
\end{aligned}
$$

The constant implicit in $\mathcal{O}$ depends on $d$, a lower bound on $\gamma_{0}$ and on upper bounds for $\|\gamma\|_{C^{1,1}(\bar{D})}$ and $\|\phi\|_{C^{1,1}\left(\mathbb{R}^{d-1}\right)}$.

Proof. By the definition of $a_{M, N}$, the term to be computed equals

$$
\begin{aligned}
N \int_{\tilde{D}} \frac{\nabla \gamma(F(x))}{\gamma(F(x))} \cdot\left(\nabla F^{-1}(F(x))^{t}\left(i \xi-e_{d}\right)\right) \chi(M x)^{2} e^{-2 N x_{d}} d x \\
\quad+M \int_{\tilde{D}} \frac{\nabla \gamma(F(x))}{\gamma(F(x))} \cdot\left(\nabla F^{-1}(F(x))^{t} \nabla \chi(M x)\right) \chi(M x) e^{-2 N x_{d}} d x .
\end{aligned}
$$

The last of these two addends is $\mathcal{O}\left(M^{2-d} N^{-1}\right)$. The first of them is analysed according to the following decomposition

$$
\begin{aligned}
\nabla F^{-1}(F(x)) \frac{\nabla \gamma(F(x))}{\gamma(F(x))}= & \nabla F^{-1}(P) \frac{\nabla \gamma(P)}{\gamma(P)}+\left(\nabla F^{-1}(F(x))-\nabla F^{-1}(P)\right) \frac{\nabla \gamma(P)}{\gamma(P)} \\
& +\nabla F^{-1}(F(x))\left(\frac{\nabla \gamma(F(x))}{\gamma(F(x))}-\frac{\nabla \gamma(P)}{\gamma(P)}\right) .
\end{aligned}
$$


The first term yields

$$
N \frac{\nabla \gamma(P)}{\gamma(P)} \cdot\left(\nabla F^{-1}(P)^{t}\left(i \xi-e_{d}\right)\right) \int_{\tilde{D}} \chi(M x)^{2} e^{-2 N x_{d}} d x
$$

which is easily computed by using that

$$
\begin{aligned}
\int_{\tilde{D}} \chi(M x)^{2} e^{-2 N x_{d}} d x & \\
& =M^{1-d} \int_{\mathbb{R}^{d-1}} \eta\left(\left|x^{\prime}\right|\right)^{2} d x^{\prime}\left(\frac{1}{2 N}+\int_{0}^{\infty}\left(\eta\left(M x_{d}\right)-1\right) e^{-2 N x_{d}} d x_{d}\right) .
\end{aligned}
$$

This already provides the leading term in the asymptotic identity stated in the lemma. We are now left with the second and third terms on the previous decomposition. For the second of them, we just need to use that

$$
\left|\nabla F^{-1}(F(x))-\nabla F^{-1}(F(0))\right| \lesssim\left|\nabla \phi\left(x^{\prime}+p^{\prime}\right)-\nabla \phi\left(p^{\prime}\right)\right|,
$$

which yields a term of the order

$$
\mathcal{O}\left(\int_{\left|x^{\prime}\right| \leq M^{-1}}\left|\nabla \phi\left(x^{\prime}+p^{\prime}\right)-\nabla \phi\left(p^{\prime}\right)\right| d x^{\prime}\right)=\mathcal{O}\left(M^{-d}\right) .
$$

Eventually, for the third term arising in the decomposition, we use that

$$
|\nabla \gamma(F(x))-\nabla \gamma(F(0))| \lesssim|x|,
$$

which yields a term of the order $\mathcal{O}\left(M^{-d}\right)$. This ends the proof of this lemma.

Now we need a lemma similar to the 2.2 but for harmonic functions. In this case, the result was proved by Brown and Salo (the lemma 2.5 in [8]):

Lemma 3.3 (Brown-Salo [8]). Assume $\phi \in C^{1,1}\left(\mathbb{R}^{d-1}\right)$. Consider $a_{M, N}$ as in (6) with $M=N^{1 / 2}$. Let $z_{M, N}$ solve the boundary value problem

$$
\left\{\begin{aligned}
\nabla \cdot\left(A \nabla z_{M, N}\right) & =-\nabla \cdot\left(A \nabla a_{M, N}\right) \text { in } \tilde{D}, \\
\left.z_{M, N}\right|_{\partial \tilde{D}} & =0,
\end{aligned}\right.
$$

where $A(x)=\nabla F^{-1}(F(x)) \nabla F^{-1}(F(x))^{t}$. Then

$$
\left\|z_{M, N}\right\|_{L^{2}(\tilde{D})} \lesssim N^{-\frac{1}{2}} M^{\frac{1-d}{2}}
$$

where the implicit constant depends on $d$ and on upper bounds for $\|\phi\|_{C^{1,1}\left(\mathbb{R}^{d-1}\right)}$.

For the choice $M=N^{1 / 2}$, we consider the functions $u_{N}$ and $v_{N}$ defined by

$$
\begin{aligned}
& F^{*} u_{N}=M^{\frac{d-1}{2}} C_{P}^{\prime}\left(a_{M, N}+w_{M, N}\right) \\
& F^{*} v_{N}=M^{\frac{d-1}{2}} C_{P}^{\prime}\left(a_{M, N}+z_{M, N}\right)
\end{aligned}
$$

with $C_{P}^{\prime}=\sqrt{2}\left(1+\left|\nabla \phi\left(p^{\prime}\right)\right|^{2}\right)^{-1 / 4}\left(\int_{\mathbb{R}^{d-1}} \eta\left(\left|x^{\prime}\right|\right)^{2} d x^{\prime}\right)^{-1 / 2}$. Let $f_{N}$ denote $\left.u_{N}\right|_{\partial D}=$ $\left.v_{N}\right|_{\partial D}$ and plug them in the right hand side of 14

$$
\int_{D} \frac{\nabla \gamma}{\gamma} \cdot \nabla u_{N} \overline{v_{N}}=\int_{D} \frac{\nabla \gamma(F(x))}{\gamma(F(x))} \cdot\left(\nabla F^{-1}(F(x))^{t} \nabla F^{*} u_{N}(x)\right) \overline{F^{*} v_{N}(x)} d x .
$$

By the identity (14) and the lemma 3.2 we have that

$$
\begin{aligned}
\int_{\partial D}\left(\frac{1}{\gamma} \Lambda_{\gamma}-\Lambda\right) f_{N} \overline{f_{N}}= & -\frac{\nabla \gamma(P)}{\gamma(P)} \cdot \frac{\nabla F^{-1}(P)^{t}\left(i \xi-e_{d}\right)}{\left(1+\left|\nabla \phi\left(p^{\prime}\right)\right|^{2}\right)^{1 / 2}}+\mathcal{O}\left(M^{-\theta}\right) \\
& +\mathcal{O}\left(M^{d-1}\left\|\delta \nabla a_{M, N}\right\|_{L^{2}(\tilde{D})}\left\|z_{M, N} / \delta\right\|_{L^{2}(\tilde{D})}\right) \\
& +\mathcal{O}\left(M^{d-1}\left\|\nabla w_{M, N}\right\|_{L^{2}(\tilde{D})}\left\|a_{M, N}+z_{M, N}\right\|_{L^{2}(\tilde{D})}\right),
\end{aligned}
$$


where $\delta(x)$ denotes the distance between $x$ and $\partial \tilde{D}$. As Brown did in [6], we use Hardy's inequality to bound

$$
\left\|z_{M, N} / \delta\right\|_{L^{2}(\tilde{D})} \lesssim\left\|\nabla z_{M, N}\right\|_{L^{2}(\tilde{D})} .
$$

The terms $\left\|\nabla z_{M, N}\right\|_{L^{2}(\tilde{D})}$ and $\left\|\nabla w_{M, N}\right\|_{L^{2}(\tilde{D})}$ can be bounded according to the lemma 2.2 For $\left\|z_{M, N}\right\|_{L^{2}(\tilde{D})}$ we will use the lemma 3.3 . The remaining terms will be bounded as follows:

Lemma 3.4. Let the function $\phi \in C^{1,1}\left(\mathbb{R}^{d-1}\right)$ and $M=N^{1 / 2}$. Then, we have that $\left\|a_{M, N}\right\|_{L^{2}(\tilde{D})}=\mathcal{O}\left(M^{(1-d) / 2} N^{-1 / 2}\right)$ and $\left\|\delta \nabla a_{M, N}\right\|_{L^{2}(\tilde{D})}=\mathcal{O}\left(M^{(1-d) / 2} N^{-1 / 2}\right)$.

Proof. We only consider $\left\|\delta \nabla a_{M, N}\right\|_{L^{2}(\tilde{D})}$, the other is a straightforward computation. It is enough to note that

$$
\begin{aligned}
\left\|\delta \nabla a_{M, N}\right\|_{L^{2}(\tilde{D})} \lesssim & N\left(\int_{\tilde{D}} \chi(M x)^{2} e^{-2 N x_{d}} x_{d}^{2} d x\right)^{1 / 2} \\
& +M\left(\int_{\tilde{D}}|\nabla \chi(M x)| \chi(M x) e^{-2 N x_{d}} x_{d}^{2} d x\right)^{1 / 2}
\end{aligned}
$$

and estimate the first of these integrals, which is the one of highest order.

These considerations, together with (3), yield the asymptotic equality

$$
\begin{aligned}
\mathcal{N}_{\gamma}\left(f_{N}, \overline{f_{N}} / \gamma\right)-\int_{\partial D} \Lambda f_{N} \overline{f_{N}}= & -\frac{\nabla \gamma(P)}{\gamma(P)} \cdot \frac{\nabla F^{-1}(P)^{t}\left(i \xi-e_{d}\right)}{\left(1+\left|\nabla \phi\left(p^{\prime}\right)\right|^{2}\right)^{1 / 2}} \\
& +\sum_{\alpha \in \mathbb{N}^{2}}\left(f_{N} \mid e_{\alpha_{1}}\right)\left(\overline{f_{N}} / \gamma \mid e_{\alpha_{2}}\right) X_{\alpha}+\mathcal{O}\left(N^{-\frac{1}{2}}\right) .
\end{aligned}
$$

As in the section 2, we need to filter out the noise in 15 to be able to recover $\left.\partial_{\nu} \gamma\right|_{\partial D}$. The situation here is a bit more involved, since

$$
\left\|f_{N}\right\|_{L^{2}(\partial D)}=\mathcal{O}(1) \text {. }
$$

However, averaging in the parameter $M=N^{1 / 2}$ we are able to get rid of the noise.

Lemma 3.5. We have that

$$
\mathbb{E}\left|\frac{1}{T} \int_{T}^{2 T} \sum_{\alpha \in \mathbb{N}^{2}}\left(f_{t^{2}} \mid e_{\alpha_{1}}\right)\left(\overline{f_{t^{2}}} / \gamma \mid e_{\alpha_{2}}\right) X_{\alpha} d t\right|^{2} \leq \frac{C}{T^{2 / 3}} .
$$

The constant $C>0$ depends on $d$, a lower bound for $\gamma_{0}$ and upper bounds for $\|\gamma\|_{C^{1,1}(\bar{D})}$ and $\|\phi\|_{C^{1,1}\left(\mathbb{R}^{d-1}\right)}$.

Proof. Start by noting that

$$
\mathbb{E}\left|\frac{1}{T} \int_{T}^{2 T} \sum_{\alpha \in \mathbb{N}^{2}}\left(f_{t^{2}} \mid e_{\alpha_{1}}\right)\left(\overline{f_{t^{2}}} / \gamma \mid e_{\alpha_{2}}\right) X_{\alpha} d t\right|^{2}=\frac{1}{T^{2}} \int_{Q_{T}}\left(f_{t^{2}} \mid f_{s^{2}}\right)\left(f_{s^{2}} / \gamma \mid f_{t^{2}} / \gamma\right) d(t, s)
$$

where $Q_{T}=[T, 2 T] \times[T, 2 T]$. Consider $S \in(0, T / 2)$ to be chosen later and set

$$
\begin{aligned}
D(S) & =\left\{(t, s) \in Q_{T}: t-S \leq s \leq t+S\right\}, \\
L(S) & =\left\{(t, s) \in Q_{T}: T \leq s<t-S\right\}, \\
R(S) & =\left\{(t, s) \in Q_{T}: t+S<s \leq 2 T\right\} .
\end{aligned}
$$

A direct computation shows that $\left|\left(f_{t^{2}} \mid f_{s^{2}}\right)\right|+\left|\left(f_{s^{2}} / \gamma \mid f_{t^{2}} / \gamma\right)\right| \lesssim 1$, and consequently,

$$
\frac{1}{T^{2}} \int_{D(S)}\left(f_{t^{2}} \mid f_{s^{2}}\right)\left(f_{s^{2}} / \gamma \mid f_{t^{2}} / \gamma\right) d(t, s) \lesssim \frac{S}{T}
$$


On the other hand, using that $\left(-i \xi^{\prime} \cdot \nabla\right) e^{i\left(t^{2}-s^{2}\right) \xi^{\prime} \cdot x^{\prime}}=\left|\xi^{\prime}\right|^{2}\left(t^{2}-s^{2}\right) e^{i\left(t^{2}-s^{2}\right) \xi^{\prime} \cdot x^{\prime}}$, where $\xi=\left(\xi^{\prime}, \xi_{d}\right)$, integrating by parts and using the regularity for $\phi$ and $\gamma$ we can see that, whenever $t \neq s$,

$$
\left|\left(f_{t^{2}} \mid f_{s^{2}}\right)\right|+\left|\left(f_{s^{2}} / \gamma \mid f_{t^{2}} / \gamma\right)\right| \lesssim \frac{t+s+1}{\left|t^{2}-s^{2}\right|}
$$

The fact that $\left|\xi^{\prime}\right| \neq 0$ justifies the integration by parts. Thus, we can bound

$$
\frac{1}{T^{2}} \int_{R(S)}\left(f_{t^{2}} \mid f_{s^{2}}\right)\left(f_{s^{2}} / \gamma \mid f_{t^{2}} / \gamma\right) d(t, s) \lesssim \frac{1}{S^{2}}
$$

where we have used that if $(t, s) \in R(S)$, then $s^{2}-t^{2} \geq(t+S)^{2}-t^{2} \geq S t>0$. The same bounds hold for the integration on $L(S)$. Finally, we choose $S$ to satisfy $S T^{-1}=S^{-2}$ and get bound claim in the statement.

As a consequence of the first part of the lemma 2.5 (with $\lambda_{N}=N^{-\theta}$ and $\theta \in$ $(0,1))$ we have that

$$
\frac{1}{T_{N}} \int_{T_{N}}^{2 T_{N}} \sum_{\alpha \in \mathbb{N}^{2}}\left(f_{t^{2}} \mid e_{\alpha_{1}}\right)\left(\overline{f_{t^{2}}} / \gamma \mid e_{\alpha_{2}}\right) X_{\alpha} d t \longrightarrow 0
$$

almost surely as $N \in \mathbb{N} \backslash\{0\}$. Recall that $T_{N}=N^{3+3 \theta / 2}$.

Proof of the theorem 3. Consider $\nu_{P}$ the unit normal vector to $\partial D$ at $P$ pointing outward and $\tau_{P}$ any unitary tangential vector at $P$. Let $\xi$ satisfy

$$
\tau_{P}=-\frac{\nabla F^{-1}(P)^{t} \xi}{\left(1+\left|\nabla \phi\left(p^{\prime}\right)\right|^{2}\right)^{1 / 2}} .
$$

Since

$$
\nu_{P}=\frac{\nabla F^{-1}(P)^{t} e_{d}}{\left(1+\left|\nabla \phi\left(p^{\prime}\right)\right|^{2}\right)^{1 / 2}}
$$

we know that $\xi \cdot A_{\gamma}(0) \xi=e_{d} \cdot A_{\gamma}(0) e_{d}, \xi \cdot A_{\gamma}(0) e_{d}=0$ and $\xi=\left(\xi^{\prime}, \xi_{d}\right)$ satisfies $\xi^{\prime} \neq 0$. In this case, 15

$$
\begin{aligned}
\mathcal{N}_{\gamma}\left(f_{t^{2}}, \overline{f_{t^{2}}} / \gamma\right)-\int_{\partial D} \Lambda f_{t^{2}} \overline{f_{t^{2}}}= & \frac{\partial_{\nu_{P}} \gamma(P)+i \tau_{P} \cdot \nabla \gamma(P)}{\gamma(P)} \\
& +\sum_{\alpha \in \mathbb{N}^{2}}\left(f_{t^{2}} \mid e_{\alpha_{1}}\right)\left(\overline{f_{t^{2}}} / \gamma \mid e_{\alpha_{2}}\right) X_{\alpha}+\mathcal{O}\left(t^{-1}\right) .
\end{aligned}
$$

The proof of the theorem ends just taking average in the interval $\left(T_{N}, 2 T_{N}\right)$ for every term of the previous asymptotic identity and using 16 .

Proof of the theorem 4. Noting that

$$
\mathbb{E}\left|\frac{1}{T_{N}} \int_{T_{N}}^{2 T_{N}} \sum_{\alpha \in \mathbb{N}^{2}}\left(f_{t^{2}} \mid e_{\alpha_{1}}\right)\left(\overline{f_{t^{2}}} / \gamma \mid e_{\alpha_{2}}\right) X_{\alpha} d t\right|^{2} \leq \frac{C}{N^{2+\theta}}
$$

with $C$ as in the lemma 3.5 we apply the second part of the lemma 2.5 for the sequence of random variables

$$
\left\{\frac{1}{T_{N}} \int_{T_{N}}^{2 T_{N}} \sum_{\alpha \in \mathbb{N}^{2}}\left(f_{t^{2}} \mid e_{\alpha_{1}}\right)\left(\overline{f_{t^{2}}} / \gamma \mid e_{\alpha_{2}}\right) X_{\alpha} d t: N \in \mathbb{N} \backslash\{0\}\right\}
$$

and $\lambda_{N}=N^{-\theta}$. Thus, we have that

$$
\mathbb{P}\left\{\left|\frac{1}{T_{N}} \int_{T_{N}}^{2 T_{N}} \sum_{\alpha \in \mathbb{N}^{2}}\left(f_{t^{2}} \mid e_{\alpha_{1}}\right)\left(\overline{f_{t^{2}}} / \gamma \mid e_{\alpha_{2}}\right) X_{\alpha} d t\right| \leq N^{-\theta}\right\} \geq 1-\epsilon
$$


for all $N \geq N_{0}$. According to 2.6 it is enough to choose $N_{0}$ satisfying

$$
\sum_{N=N_{0}}^{\infty} \frac{C}{N^{2-\theta}}<\epsilon
$$

with $C$ as in the lemma 3.5 , which holds whenever

$$
\left(N_{0}-1\right)^{1-\theta}>\frac{\epsilon^{-1}}{C(1-\theta)} \text {. }
$$

Since

$$
\begin{aligned}
\left\{\mid \frac{1}{T_{N}} \int_{T_{N}}^{2 T_{N}} \sum_{\alpha \in \mathbb{N}^{2}}\right. & \left.\left(f_{t^{2}} \mid e_{\alpha_{1}}\right)\left(\overline{f_{t^{2}}} / \gamma \mid e_{\alpha_{2}}\right) X_{\alpha} d t \mid \leq N^{-\theta}\right\} \\
& \subset\left\{\left|Y_{N}-\frac{\partial_{\nu_{P}} \gamma(P)+i \tau_{P} \cdot \nabla \gamma(P)}{\gamma(P)}\right| \leq C N^{-\theta}\right\},
\end{aligned}
$$

we can conclude the inequality stated in the theorem.

Acknowledgements. The authors are partially supported by BERC 2014-2017 and the MINECO grant BCAM Severo Ochoa SEV-2013-0323. PC is also supported by the MINECO project MTM2015-69992-R, and would like to thank Ikerbasque - Basque Foundation for Science for their support and encouragement. AG is also supported by the MINECO project MTM2014-53145-P.

\section{REFERENCES}

[1] Giovanni Alessandrini. Stable determinations of conductivity by boundary measurements. Appl. Anal., 27(1-3):153-172, 1988.

[2] Giovanni Alessandrini. Singular solutions of elliptic equations and the determination of conductivity by boundary measurements. J. Differ. Equations, 84(2):252-272, 1990.

[3] Kari Astala and Lassi Päivärinta. Calderón's inverse conductivity problem in the plane. Ann. Math. (2), 163(1):265-299, 2006.

[4] Juan Antonio Barceló, Tomeu Barceló, and Alberto Ruiz. Stability of the inverse conductivity problem in the plane for less regular conductivities. J. Differ. Equations, 173(2):231-270, 2001.

[5] Tomeu Barceló, Daniel Faraco, and Alberto Ruiz. Stability of Calderón inverse conductivity problem in the plane. J. Math. Pures Appl. (9), 88(6):522-556, 2007.

[6] R.M. Brown. Recovering the conductivity at the boundary from the Dirichlet to Neumann map: A pointwise result. J. Inverse Ill-Posed Probl., 9(6):567-574, 2001.

[7] Russell M. Brown. Global uniqueness in the impedance-imaging problem for less regular conductivities. SIAM J. Math. Anal., 27(4):1049-1056, 1996.

[8] Russell M. Brown and Mikko Salo. Identifiability at the boundary for first-order terms. Appl. Anal., 85(6-7):735-749, 2006.

[9] Russell M. Brown and Rodolfo H. Torres. Uniqueness in the inverse conductivity problem for conductivities with $3 / 2$ derivatives in $L^{p}, p>2 n$. J. Fourier Anal. Appl., 9(6):563-574, 2003.

[10] Russell M. Brown and Gunther A. Uhlmann. Uniqueness in the inverse conductivity problem for nonsmooth conductivities in two dimensions. Commun. Partial Differ. Equations, 22(56):1009-1027, 1997.

[11] Alberto P. Calderón. On an inverse boundary value problem. Comput. Appl. Math., 25(23):133-138, 2006

[12] Pedro Caro, Andoni García, and Juan Manuel Reyes. Stability of the Calderón problem for less regular conductivities. J. Differ. Equations, 254(2):469-492, 2013.

[13] Pedro Caro and Keith M. Rogers. Global uniqueness for the Calderón problem with Lipschitz conductivities. Forum Math. Pi, 4:28, 2016.

[14] Albert Clop, Daniel Faraco, and Alberto Ruiz. Stability of Calderón's inverse conductivity problem in the plane for discontinuous conductivities. Inverse Probl. Imaging, 4(1):49-91, 2010.

[15] Matthew M. Dunlop and Andrew M. Stuart. The Bayesian formulation of EIT: analysis and algorithms. Inverse Probl. Imaging, 10(4):1007-1036, 2016.

[16] J.N. Franklin. Well-posed stochastic extensions of ill-posed linear problems. J. Math. Anal. Appl., 31:682-716, 1970. 
[17] Andoni García and Guo Zhang. Reconstruction from boundary measurements for less regular conductivities. Inverse Problems, 32(11):115015, 2016

[18] Boaz Haberman. Uniqueness in Calderón's problem for conductivities with unbounded gradient. Commun. Math. Phys., 340(2):639-659, 2015.

[19] Boaz Haberman and Daniel Tataru. Uniqueness in Calderón's problem with Lipschitz conductivities. Duke Math. J., 162(3):497-516, 2013.

[20] Hanne Kekkonen, Matti Lassas, and Samuli Siltanen. Analysis of regularized inversion of data corrupted by white Gaussian noise. Inverse Probl., 30(4):18, 2014.

[21] Hanne Kekkonen, Matti Lassas, and Samuli Siltanen. Corrigendum to: "Analysis of regularized inversion of data corrupted by white Gaussian noise". Inverse Probl., 32(9):3, 2016

[22] Kim Knudsen, Matti Lassas, Jennifer L. Mueller, and Samuli Siltanen. Regularized D-bar method for the inverse conductivity problem. Inverse Probl. Imaging, 3(4):599-624, 2009.

[23] Robert V. Kohn and Michael Vogelius. Determining conductivity by boundary measurements. Commun. Pure Appl. Math., 37:289-298, 1984.

[24] Robert V. Kohn and Michael Vogelius. Determining conductivity by boundary measurements. II: Interior results. Commun. Pure Appl. Math., 38:643-667, 1985.

[25] Adrian I. Nachman. Reconstructions from boundary measurements. Ann. Math. (2), 128(3):531-576, 1988.

[26] Adrian I. Nachman. Global uniqueness for a two-dimensional inverse boundary value problem. Ann. Math. (2), 143(1):71-96, 1996.

[27] Lassi Päivärinta, Alexander Panchenko, and Gunther Uhlmann. Complex geometrical optics solutions for Lipschitz conductivities. Rev. Mat. Iberoam., 19(1):57-72, 2003.

[28] V.N. Sudakov and L.A. Halfin. Statistical approach to the correctness of problems in mathematical physics. Sov. Math., Dokl., 5:1094-1096, 1964.

[29] John Sylvester and Gunther Uhlmann. A global uniqueness theorem for an inverse boundary value problem. Ann. Math. (2), 125:153-169, 1987.

[30] John Sylvester and Gunther Uhlmann. Inverse boundary value problems at the boundarycontinuous dependence. Commun. Pure Appl. Math., 41(2):188-219, 1988.

[31] A.N. Tikhonov. On the stability of inverse problems. C. R. (Dokl.) Acad. Sci. URSS, n. Ser., 39:176-179, 1943.

[32] A.N. Tikhonov. Solution of incorrectly formulated problems and the regularization method. Sov. Math., Dokl., 5:1035-1038, 1963.

BCAM - Basque Center for Applied mathematics

E-mail address: pcaro@bcamath.org

E-mail address: agarcia@bcamath.org 